\title{
A hedgehog-like appearance resulting from Hari acupuncture
}

\author{
Seong-Mi Park MD PhD, Wan-Joo Shim MD PhD
}

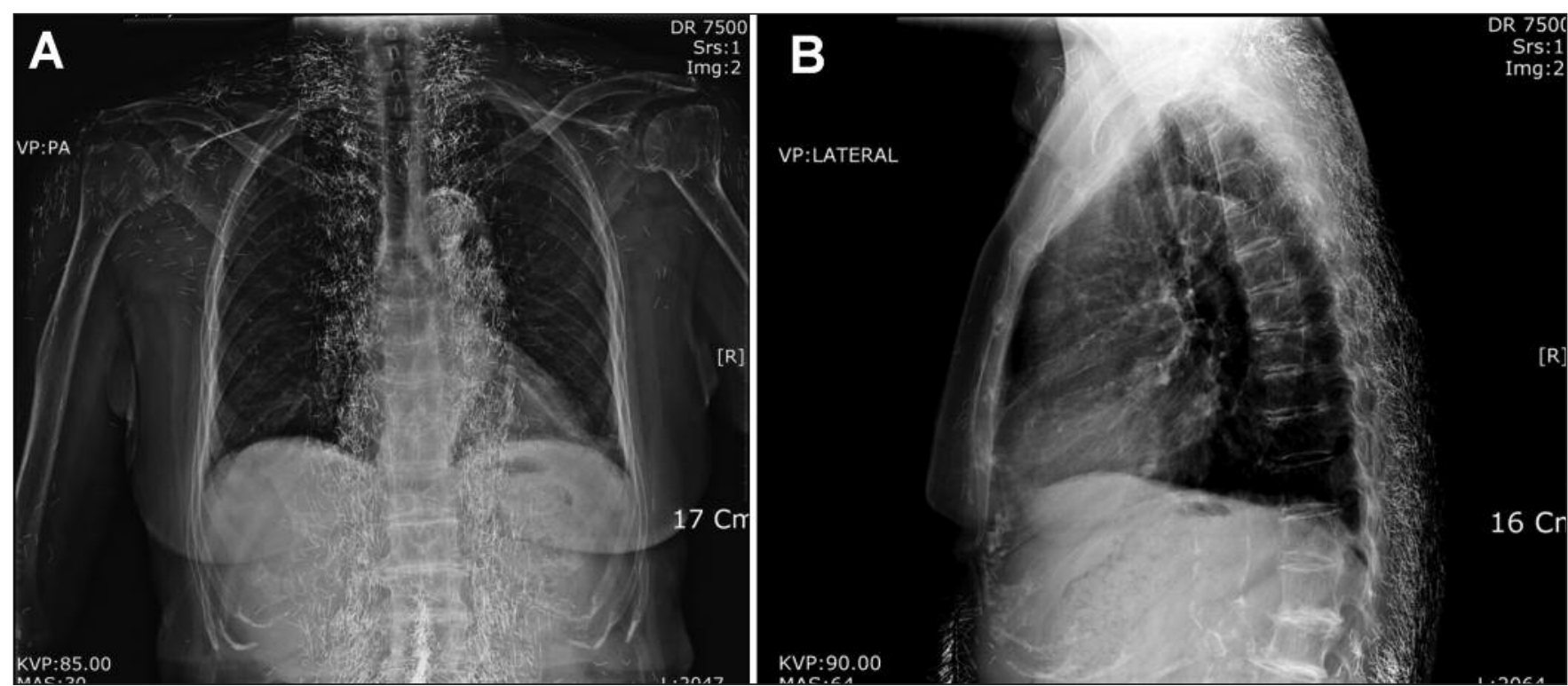

Figure 1: (A) Plain radiograph of the chest of an 89-year-old woman showing countless subcutaneous needle fragments over her neck and chest. (B) Lateral radiograph of the chest showing a striking hedgehog-like appearance.

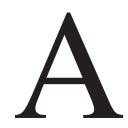
n 89-year-old woman with a history of osteopenia and multiple spinal compression fractures presented with an exacerbation of her chronic back and joint pain, a month after she slipped on the stairs. Radiographs showed countless foreign bodies along her neck, chest (Figure 1A), abdomen and pelvis, and her lateral chest radiograph bore a striking hedgehog-like appearance (Figure 1B). Despite this, examination of our patient's skin was unremarkable. She had received gold-needle acupuncture therapy on many occasions over several years to relieve pain in her "whole body." The opacities seen in the radiographs represent indwelling gold acupuncture needles aligned along vertical meridians of the torso.

Traditional Chinese acupuncture involves placing needles into the subcutaneous tissues along predefined meridians and, later, totally removing the needles. A unique subtype of acupuncture known as Hari involves the permanent placement of fine needles into the subcutaneous tissues; this form of acupuncture is not uncommon in Northeast Asia. The retained needles are typically 3 to $15 \mathrm{~mm}$ in length and $1 \mathrm{~mm}$ in diameter, and they may appear straight, curved or semicircular. ${ }^{1}$

Traditional acupuncture has generally been considered a safe procedure; however, complications have been reported, including infection, pneumothorax, spinal cord injury and cardiac tamponade. ${ }^{2}$ Although a similar range of complications has been described for Hari, its relative safety compared with traditional Chinese acupuncture is unknown. ${ }^{3}$

\section{References}

1. Vassiou K, Kelekis NL, Fezoulidis IV. Multiple retained acupuncture needle fragments. Eur Radiol 2003;13:1188-9.

2. White A. A cumulative review of the range and incidence of significant adverse events associated with acupuncture. Acupunct Med 2004;22:122-33.

3. Yamashita H, Tsukayama H, White AR, et al. Systematic review of adverse events following acupuncture: the Japanese literature. Complement Ther Med 2001;9:98-104.
Competing interests: None declared.

This article has been peer reviewed.

Correspondence to: Dr. Wan-Joo Shim, wjshimmd@unitel.co.kr

Affiliations: From the Department of Internal Medicine, Korea University Anam Hospital, Korea University College of Medicine, Seoul, Korea

CMAJ 2011. DOI:10.1503 /cmaj.110128 